placed on optical methods of measurement. Besides describing a simplified form of microinterferometer which he has developed, M. Mirau dealt with other optical methods for assessing the bearing area and also the quality of polish of surfaces. The complexity of the practical aspects of surface finish of mating parts was discussed in the paper by Mr. R. E. Reason (Taylor, Taylor and Hobson, Ltd.), reviewing developments and trends in this field. Mr. D. B. Ebsworth (Bristol Aeroplane Co., Ltd.) described methods employed in the production of fine surfaces to the exacting requirements of the aircraft industry. In this work a fine surface finish is required, not only to minimize hand fitting and the period of running-in, but also to reduce the risk of fatigue failure, which so often starts from minute surface defects on the highly stressed components.

On the morning of October 24 the Metrology Division of the National Physical Laboratory was opened to delegates, who were able to see the measuring equipment and methods employed at the Laboratory and also exhibits of equipment arranged by a number of the authors of papers.

H.M. Stationery Office will publish the proceedings of the conference, and these will contain the texts of the papers and a report of the associated discussions. T. R. J. OAKLEY

\section{THE POTATO ROOT EELWORM AND ITS CONTROL}

$\mathrm{T}$

HE Association of Applied Biologists held a symposium on "The Potato Root Eelworm" at the Imperial College of Science and Technology, London, on January 8, which was well attended and presented a wealth of detailed work, including a demonstration of economic control under certain conditions ; some hope for the production of potatoes resistant to the eelworm was also raised. The chairman, Dr. I. Thomas, outlined the magnitude of the problem. Although it is seventy-three years since eelworm cysts were found on potato roots, it was not until 1913 that severe disease was reported from Scotland and Rostock, and the organism was afterwards named Heterodera rostochiensis. Since that time, the disease has spread to many parts of the world, half a million acres of arable land being affected in England alone during 1949. About 150,000 acres of potatoes are attacked annually, onethird seriously, giving an overall loss of some 200,000 tons of potatoes worth $£ 2,000,000$. In advisory work alone, questions of potato root eelworm absorb a major share of the activities of 80-100 workers who spend about 40 per cent of their time on nematological problems. This last statement by Dr. Thomas seemed, more than any other, to impress members of the conference with the serious nature of the problem.

The morning session was devoted to papers on the hatching of eggs from cysts, and on progress in breeding potatoes resistant to the eelworm. It is vital, in any experimental work with potato root eelworm, to know how many of the contents of the resistant, 'dormant' cyst are still potentially active, and this can be measured by treating the cysts with 'potato root diffusate'- the drainage from potatoes grown in sand culture. Eggs within the cyst hatch into larval eelworms under the stimulus of this liquid, just as they would when potatoes are grown on eelworm-infested land. Dr. D. W. Fenwick gave the results of his extensive studies of this laboratory measurement. When the numbers of larvæ emerging from the cyst are plotted against the logarithm of time in days, a symmetrical sigmoid curve is obtained. Application of probit technique gives a linear relation. Dr. Fenwick then proceeded to show that, since the total number of larvæ emerging is twice the number obtained at the point of inflexion of the sigmoidal curve, much time and space can be saved in laboratory determinations, for numbers only need to be estimated for a time sufficient to establish the point of inflexion. The slope of the probit line is a measure of the susceptibility of cyst contents to root diffusate. Practical findings checked by these theoretical considerations include several important items : temperatures between $10^{\circ}$ and $25^{\circ} \mathrm{C}$. have little effect on the total hatch, although the rate of hatching increases with rise in temperature; hatching is virtually inhibited at $30^{\circ} \mathrm{C}$. ; pre-soaking of the cysts in water hastens hatching; $p H$ of the root diffusate has no effect between 3.0 and 8.0 ; sunlight almost inhibits hatching; hatching in a given sample is proportional to the logarithm of the concentration of the diffusate ; and since the activities of root diffusate are inhibited in steam-sterilized soil for some weeks, its action appears to be linked with the microflora.

Mr. T. G. Onions spoke on the pattern of hatching from the cyst. He has subjected cysts to the action of root diffusate for a period, and then sectioned them for microscopic examination, to see where the empty, hatched eggs lie. He found most near the vulval end of the cyst, and obtained more from the periphery than from the middle. It was thought that this pattern of hatching might reflect a gradient of oxygen tension.

It would be a great advance in control if potatoes could be bred with resistance to the potato root eelworm. Mr. F. G. W. Jones reviewed the possibilities and progress of this approach. Resistance to this particular eelworm is found in Solanum ballsii $(=$ vernii) and also in Solanum andigenum, which may be no more than a variant of $S$. tuberosum. German workers are investigating the possibilities of introducing resistance through crossing potatoes with $S$. ballsii, and Dutch and British workers are using $S$. andigenum as the resistant parent. The mechanism seems to be that of a dominant gene responsible for resistance, with tetrasomic inheritance giving the possibility of all degrees of resistance and susceptibility. Practical degrees of resistance as yet attained are rather low; but it might be useful to approach the problem of resistance through some contributory issue. Crosses between $S$. stenotomum and S. vernii, for example, have low cyst-hatching stimulus which may add, in practice, to their resistance. Another possible line of approach would be to combine high cyst-hatching stimulus with innate resistance, in order to provide the ideal trap crop, and thereby reduce the effective eelworm population.

The afternoon session on direct control opened with a paper by Dr. B. G. Peters on "Laboratory and Pot Tests of Nematicides". These tests are a first stage in assessment of the value of any material for such control. A certain amount of knowledge can be obtained quickly by dispersing a toxicant under test in water and inoculating it with the vinegar eelworm in a special double-flask culture apparatus. Dr. Peters also described the necessary controls and measurements to obtain the maximum 
information from pot experiments-to make allowance for natural death, variations in eelworm population, and other factors. He has, indeed, reduced the technique of such tests to a process as exact as possible, and gave examples of their use with D.D. mixture (dichloropropane-dichloropropylene), ethylene dibromide and other materials. A typical useful practical result from pot experiments is that a single injection with D.D. gave a percentage kill of larvæ superior to that with two or three consecutive injections.

Dr. J. Grainger described an economic field control with D.D. on first-early 'Epicure' potato crops in Ayrshire. Increases in yield in numerous experiments since 1947 have been worth about twice the cost of treatment, whether applied at $200 \mathrm{lb}$. or $400 \mathrm{lb}$. an acre. Later-lifted crops do not command such high prices a ton as first-earlies, and the yield increase only equals the cost of treatment. Evidence was given for the belief that D.D. has a three-fold action: it can diminish the number of larvæ within cysts; it can diminish the number of full eggs in the eyst in the absence of potatoes; and it can stimulate growth of the potato crop directly. Long-term experiments on possible control of potato root eelworm on main-crops grown in a seven-year rotation were described. They are designed to make the best use of the three-fold action, which also supplies the reason for treating first- and second-early crops as long as possible before the potatoes are planted. Dr. Grainger also briefly described his 'Auchincruive' soil injectors for the application of D.D.; these are now in commercial production. Several results indicated that, for equal quantities an acre, the performance of D.D. was superior when injected as 'spots' at 15-in. centres rather than in continuous traces $12 \mathrm{in}$. apart. Other materials showing sufficient promise to be investigated on a field scale are ethylene dibromide and inorganic compounds of mercury. These both appear to lessen the build-up of the eelworm population when potatoes are grown, and mercury compounds would be much cheaper than D.D. if they could be mixed finely enough with the soil.

An interesting study on possible control was described by Mr. L. N. Staniland. Phenols, cresols and xylenols and their chlorinated forms give some control of potato root eelworm ; but their performance is greatly improved by 'solubilization' with detergents, preferably of the long-chain alkyl sulphate type. They are used as soil drenches at 1 gallon/sq. ft., and at present are quite uneconomic for field application. In the glasshouse, their use is cheaper than steam sterilization, but dearer than D.D. There is, moreover, a danger of tainting the crop. In spite of all these present economic handicaps, however, Mr. Staniland described ways in which performance could possibly be improved. Lower concentrations with more detergent, the introduction of small amounts of iodine, and the performance of different chlorinated compounds are all under test, and Mr. Staniland's audience felt that whether the study ultimately developed economically or not, it would be a sound contribution to the problem.

Prof. H. W. Miles opened the discussion on the morning session of the conference. He said that eelworm study was somewhat neglected in the past, and that the approach to the problem first arose from the farmer. Host-parasite relations are most important for a correct understanding of the problem, and laboratory work is beginning to equal that devoted to the practical approach. Discussion during the afternoon session was initiated by Dr. W. Heeley, who thanked the Association for the opportunities afforded by the symposium, and summarized the economic considerations of direct control arising from the lectures. The use of D.D., he said, comes nearest to practical control at present. It has been suggested that its action might in practice be indirect, putting host and parasite somewhat out of phase. Interesting matters arising from the general discussion are that the breakdown of cysts is slower in peat soils than in loam (Dr. Fenwick), and that potato root eelworm has been found in Peru, one of the native homes of the resistant Solanum species. These species are, however, less resistant to the Peruvian strain than to the European eelworm (Dr. C. Ellenby). The question was also raised as to whether a seven-year rotation would itself control infestation of potato root eelworm in practice. Dr. Grainger maintained that the period is not long enough, and certainly not in west Scotland. Infestations capable of causing disease can persist there for 20-25 years in the absence of potatoes. Such results are to be expected by calculation from experiments on the rate of natural loss, and have been confirmed by authenticated advisory cases.

\section{THE RUBBER RESEARCH INSTITUTE OF MALAYA: BOTANICAL DIVISION}

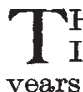

HE botanical work of the Rubber Research Institute of Malaya, Kuala Lampur, for the years 1949-51, as presented in its report, is by way of being an interim account of the five-year plan begun in 1949. Important new developments and discoveries, relating to the extensive investigations now in progress, are briefly indicated under the headings of breeding and selection, propagation and planting techniques, tapping, physiology, and ecology. The programme on breeding and selection deals with such items as pollination, the large-scale production of improved seedlings, cytogenetical studies of Hevea, the examination of criteria for selection in the laboratory and in the field, the selection of individuals and clones based on their vegetative characters, the quality and properties of latex and rubber, and selection for resistance to disease, etc.

Studies of propagation and planting technique have related to such matters as the effectiveness 0 bud-grafting on to mature root systems, the prebudding treatment of budwood, the storage of Hevea seeds, stock-scion relationships, density of planting and distribution, the effect of selective thinning-out, the value of mixed planting of Hevea and other crops, for example, cacao, and methods of new planting and replanting. Work on tapping has been concerned with tapping systems for seedling and budded trees and slaughter tapping experiments. Physiological studies of the nutrition of Hevea have been continued, attention being paid to the cause of the chronic defoliation in certain clones and to latex flow in response to tapping. Researches of a more fundamental character on the physiology of latex are also in progress. The effects of various injected substances, for example, copper salts, on the yield and properties of latex, and the stimulation of latex 